OPEN ACCESS

Edited by:

Xavier Noel,

Free University of Brussels, Belgium

Reviewed by:

Mette Bentz,

Mental Health Services, Denmark

Antoine Bechara,

University of Southern California,

United States

*Correspondence:

Kate Tchanturia

kate.tchanturia@kcl.ac.uk

Specialty section: This article was submitted to

Psychopathology,

a section of the journa

Frontiers in Psychiatry

Received: 30 October 2018 Accepted: 27 June 2019

Published: 18 July 2019

Citation:

Sedgewick F, Kerr-Gaffney J, Leppanen J and Tchanturia K (2019) Anorexia Nervosa, Autism, and the ADOS: How Appropriate Is the New Algorithm in Identifying Cases?

Front. Psychiatry 10:507.

doi: 10.3389/fpsyt.2019.00507

\section{Anorexia Nervosa, Autism, and the ADOS: How Appropriate Is the New Algorithm in Identifying Cases?}

\author{
Felicity Sedgewick, Jess Kerr-Gaffney, Jenni Leppanen and Kate Tchanturia* \\ Eating Disorders Unit, King's College London, London, United Kingdom
}

Thirty years of scholarship has suggested that anorexia nervosa (AN) may be a "female presentation" of autism, supported by work which has found elevated rates of autism traits and diagnoses among women with clinical levels of AN. These traits are often assessed using the Autism Diagnostic Observation Schedule 2nd Edition (ADOS-2), considered the "gold-standard" tool. Recently, the authors of the ADOS-2 revised the diagnostic algorithm for the adult version of the assessment-the one most often used with AN patients. We therefore examined differences in the scores, rates of diagnosis, and correlations with other mental health issues between the two diagnostic algorithms among women with and without AN. One hundred seventy-five women with current AN, who had recovered from $A N$, and with no history of $A N$, between the ages of 12 and 53, took part in an ADOS-2 assessment. Their scores were then calculated according to both the original and the new algorithms. The new ADOS-2 algorithm identifies more women as potentially being on the autism spectrum than the old algorithm. Under both algorithms, more currently ill AN patients were identified as potentially being autistic than those with no history of AN. Recovered individuals represented a midpoint between the scores of those with and without AN on both algorithms. There were no correlations with mental health scores in any group, meaning that the new ADOS-2 algorithm is not falsely identifying anxious behaviors or depressive presentations as signs of autism in this group. Overall, we found that more AN patients and recovered individuals scored above cut-off on the new ADOS-2 algorithm, suggesting that women who experience AN may have more autistic traits, which in part persist following weight restoration and recovery. However, the ADOS-2 should not be used alone but in combination with broader clinical assessments to determine whether an autism diagnosis is appropriate for these women.

Keywords: anorexia nervosa, autism, comorbidity, women, clinical interview

\section{INTRODUCTION}

Autism is a neurodevelopmental condition which is present from early childhood, although it may not be identified until much later, especially in girls and women (1). It is associated with a range of difficulties and differences in the realms of social imagination, social communication, restricted and repetitive behaviors and interests (RRBIs), and sensory processing (2). Autistic individuals have greater cognitive rigidity than neurotypical controls $(3,4)$, showing a preference for sameness (5). Autistic people have been also shown to have a detail-oriented focus over global-oriented focus 
when undertaking visual search tasks $(6,7)$ and more generally $(8,9)$. Furthermore, difficulties in social relationships, including social imagination and social communication difficulties, are a core diagnostic feature of autism (2).

Anorexia nervosa (AN) is a complex mental health condition characterized by relentless pursuit of weight loss and extreme thinness (2). In addition to eating-related symptoms, theoretical models and empirical data suggest that social-emotional and cognitive difficulties play an important role in the development and progression of AN (10-12). Experimental findings lend support to these models demonstrating that people with AN show reduced social-emotional communication, greater attentional bias towards negative emotional cues, and poorer theory of mind relative to healthy control (HC) participants (13-16). Furthermore, people with AN have also been found to have greater detail focus at the expense of the big picture, poor cognitive flexibility, and strict rule adherence when compared to HCs (17-19). Together, these findings have led to increasing interest in the theorized association between $\mathrm{AN}$ and autism.

An association between autism and $\mathrm{AN}$ in women has been theorized since the 1980s (20) and in the decades since there has been a rapid expansion in the amount of research which supports this connection. A recent systematic review found that between 4.0 and $33.0 \%$ of people with acute AN scored above the cut-off on selfreport questionnaire measures assessing the presence of autistic features (21). The authors also found that studies using clinician or researcher administered diagnostic interview schedules, such as the Autism Diagnostic Observation Schedule 2nd Edition (ADOS2) (22), found that $4.5-53 \%$ of people with acute AN scored above the cut-off for a diagnosis of autism (21). Interestingly, only one of the included studies examined autistic features among people who had recovered from $\mathrm{AN}$ and reported that $21 \%$ of them scored above diagnostic cut-off (23). This is an interesting finding considering the impact that severe malnutrition can have on social and cognitive functioning, including social withdrawal, difficulties concentrating, and apathy (24). Taken together, there appears to be an over-representation of autism in acute AN, and further examination of presence of autism among people who have recovered from $\mathrm{AN}$ would be of interest.

Recently, the authors of the original ADOS have developed a new diagnostic algorithm for Module 4 of the schedule, which is designed to be conducted with individuals who are verbally fluent and for whom it is no longer developmentally appropriate to play with toys and figurines, i.e., older adolescents and adults (22). This follows a full revision of the other modules of the original ADOS-G (25) to create the ADOS-2 (22). The new algorithm was designed to be more sensitive to symptom severity and reflects a move to give more weight to the sensory sensitivities and sensory-motivated behaviors of autistic people, following the inclusion of sensory sensitivities in the diagnostic criteria in the Diagnostic and Statistical Manual 5th Edition (DSM-V) (2) definition of autism. However, psychomotor agitation, including tapping, restlessness, and fidgeting, may be easily confused as sensory-motivated behaviors. Therefore, as psychomotor agitation is a common feature of both anxiety and mood disorders (26), careful assessment of usefulness of the new ADOS-2 Module 4 algorithm among highly anxious and depressed individuals, including people with AN, is of interest.
Therefore, in the current study, we sought to explore the use of the new ADOS-2 Module 4 algorithm among women and adolescent girls with current $\mathrm{AN}$, those who have recovered from AN (REC), and those who have never experienced an eating disorder (HC). We expected to see higher levels of autistic traits in patients and recovered individuals than in healthy controls, both under the old and new ADOS-2 algorithm. We also expected that more possible autism cases would be identified by the new algorithm than the old algorithm in all groups. Further to this, we expected that current AN patients would show more autistic behaviors than recovered individuals, as there is a theory that being in a state of malnutrition may exacerbate or mimic autistic traits (24). Finally, we also explored the potential relationships between external presentation of autistic behaviors as measured by the original and new ADOS-2 algorithms and self-reported mental health as well as state of malnutrition as measured by body mass index (BMI) within the $\mathrm{AN}$ and REC groups.

\section{METHODS}

\section{Participants}

One hundred seventy-five women and girls between the ages of 12 and 53 took part in the study. Sixty-three were HC, 66 were currently ill with $\mathrm{AN}$ (AN), and 46 were recovered from AN (REC). The groups were matched in terms of IQ, and each group had a similar ethnic make-up. All AN and REC participants had clinical diagnoses of anorexia nervosa according to the DSM-V (American Psychiatric Association, 2013). Participants were recruited from a range of clinical and community sites across the UK, under ethical approval from the London Surrey Research Ethics Committee (17/LO/2071; 17/LO/1960). All participants gave written informed consent before taking part in the study, and written parental consent was obtained for participants under the age of 16. All procedures were conducted in accordance with the latest version of the Declaration of Helsinki.

\section{Measures \\ AQ-10}

The Autism Quotient-10 item version (27) is a brief 10-item screening questionnaire assessing the presence of autistic features. The questionnaire gives a maximum score of 10 , and 6 is generally used as the threshold a potential autism case.

\section{EDE-Q}

The Eating Disorder Examination Self-Report Questionnaire (28) is a 36-item self-report questionnaire assessing eating disorder psychopathology over the past 28 days.

\section{HADS}

The Hospital Anxiety and Depression Scale (29) is a 14-item self-report questionnaire assessing levels of both anxiety and depression over the past 2 weeks. Answers are summed for a maximum score of 42 . The present study used the total score to assess level of self-reported mood and anxiety symptoms. 


\section{ADOS-2}

The Autism Diagnostic Observation Schedule 2nd Edition (22) is a clinician- or researcher-administered structured observation, generally lasting from $30 \mathrm{~min}$ to an hour. It consists of a range of activities and interview questions designed to elicit behaviors and cognitions traditionally associated with autism. These visible behaviors and discussions are then scored from 0 to 3 for "autism severity," with a score of 3 on any one item reflecting that the person behaved in a highly autistic way, and a score of 0 indicating that the person behaved as would be expected for a neurotypical individual. Under the original algorithm, 11 items from the larger scoring matrix are then summed to create an ADOS score, where 7 is the cut-off for being designated as "on the autism spectrum," and 10 is the cut-off for being designated as "autistic." The new algorithm comprises two subscales: social affect (SA) and restrictive and repetitive behaviors (RRBs). For the diagnostic cut-off, the sum of the two subscales is used, with a score of 8 or more indicating an autism diagnosis.

\section{GENERAL PROCEDURE}

Participants were all seen at the Institute of Psychiatry, Psychology, and Neuroscience, King's College London. The data were collected as part of a larger study. Participants completed demographic information, the EDE-Q, and the HADS as part of online questionnaires. The larger testing session lasted $\sim 3 \mathrm{~h}$, including the ADOS-2, a range of neurocognitive tests, and a structural and functional MRI scan (reported elsewhere). The ADOS- 2 was scored immediately after the testing session by the administering researcher, and $20 \%$ of videos were double coded by other ADOS2 -trained researchers within the group, resulting in an inter-rater reliability of $87 \%$. Two videos were further included in a wider reliability meeting with members of other research groups within the institution, and the inter-rater reliability for these was $81 \%$, with group consensus codes being used in the final analysis.

\section{DATA ANALYSIS}

All data analyses were conducted with R (30). Due to differences in group sizes, the presence of significant heteroscedasticity was assessed using the Breusch-Pagan test (31-33). Where significant heteroscedasticity was present, group differences in clinical and demographic measures as well as in ADOS-2 scores were assessed using robust $\mathrm{M}$-estimator with Huber's approach for controlling for influential outliers (34). In the absence of significant heteroscedasticity, group differences were investigated with multiple linear regressions. Significant differences in the number of individuals who met the criteria for autism spectrum diagnosis under the original and new algorithms were examined using generalized linear binomial mixed model (35). Significance level was set at $p<0.05$.

Within the AN and REC groups, Spearman's correlations between ADOS-2 scores under the original and new algorithms and self-reported clinical variables including eating disorder symptomatology, depression, and anxiety, as well as BMI were explored. Correlations analyses were corrected for multiple comparisons using false discovery rate set at $q=0.05$, and $p<$ 0.009 was considered significant (36).

\section{RESULTS}

\section{Clinical and Demographic Characteristics}

The sample characteristics are presented in Table 1. As expected, there was a significant group difference in BMI, with $\mathrm{AN}$ participants having a significantly lower BMI than both $\mathrm{HC}$ and REC participants [AN vs. HC: $t(172)=-10.73, p<0.001$; AN vs. REC: $t(172)=-7.56, p<0.001$; HC vs. REC: $t(172)=2.12, p=$ 0.089]. There was also a significant difference in age between the groups such that the REC group was significantly older than the $\mathrm{HC}[t(172)=-3.07, p=0.007]$ and the AN group $[t(172)=-3.51$, $p=0.002]$. There was no significant difference in age between the $\mathrm{AN}$ and HC participants $[t(172)=-0.45, p=0.896]$. Finally, there was significant heteroscedasticity in the HADS $(\mathrm{BP}=14.78, p<$ $0.001)$, EDE-Q (BP $=10.05, p=0.007)$, and AQ-10 (BP = 9.38, $p=$ 0.009 ) total scores between the groups, and group differences were examined with a robust $\mathrm{M}$-estimator. As expected, the AN group reported significantly more eating disorder symptomatology than the HC $(z=17.21, p<0.001)$ and REC groups $(z=13.28, p<0.001)$. Interestingly, the REC group also reported significantly more eating disorder symptomatology than the HC group $(z=-2.59$, $p=0.026)$. Both AN and REC groups also reported significantly more depression and anxiety than the HC participants, with the currently ill AN group reporting more mood and anxiety symptoms than those who were recovered (AN vs. HC: $z=13.18$, $p<0.001$; AN vs. REC: $z=6.68, p<0.001$; HC vs. REC: $z=-5.50$,

TABLE 1 | Demographic and clinical information about participants by group.

\begin{tabular}{|c|c|c|c|c|}
\hline Measure & $\begin{array}{c}\text { HC } \\
\text { Mean (SD) }\end{array}$ & $\begin{array}{c}\text { AN } \\
\text { Mean (SD) }\end{array}$ & $\begin{array}{c}\text { REC } \\
\text { Mean (SD) }\end{array}$ & $F$ statistic, $p$ value \\
\hline Age & $21.48(3.95)$ & $21.14(5.64)$ & $24.95(7.43)$ & $F(2)=6.94, p=0.001$ \\
\hline $\mathrm{IQ}$ & $110.10(5.56)$ & $108.16(5.43)$ & $110.30(5.70)$ & $F(2)=1.35, p=0.27$ \\
\hline $\mathrm{BMl}$ & $21.76(2.15)$ & $17.22(2.56)$ & $21.01(2.40)$ & $F(2)=60.87, p<0.001$ \\
\hline EDE-Q total & $0.82(0.95)$ & $3.85(1.37)$ & $1.54(1.59)$ & $F(2)=86.14, p<0.001$ \\
\hline HADS total & $7.10(4.12)$ & $22.42(7.89)$ & $13.79(6.90)$ & $F(2)=75.14, p<0.001$ \\
\hline$A Q-10$ & $2.07(1.49)$ & $3.98(2.38)$ & $3.42(2.46)$ & $F(2)=12.99, p<0.001$ \\
\hline
\end{tabular}

N.B. IQ, intelligence quotient; BMI, body mass index; EDE-Q, Eating disorders examination questionnaire; HADS, hospital anxiety and depression scale; AQ-10, autism quotient (short version); HC, healthy control; $A N$, anorexia nervosa; REC, recovered from anorexia nervosa; SD, standard deviation. 
$p<0.001)$. Finally, the AN and REC groups also reported more autistic features than the HC group (AN vs. HC: $z=5.07, p<0.001$; AN vs. REC: $z=1.69, p=0.210$; HC vs. REC: $z=-2.93, p=0.009$ ).

\section{ADOS Scores \\ Original Algorithm}

The multiple linear regression showed that there was a significant effect of group on ADOS-2 score (Table 2; Figure 1). Post hoc t-tests revealed that this was driven by a significant difference between $\mathrm{AN}$ patients and $\mathrm{HC}$ participants, such that currently ill AN patients scored significantly higher on the old ADOS-2 algorithm, $t(172)=$ $3.68, p<0.001$. There was no significant difference in ADOS-2 scores between the AN and REC groups, $t(172)=1.37, p=0.359$, or the REC and HC groups, $t(172)=-1.99, p=0.118$.

\section{New Algorithm}

Due to significant heteroscedasticity, group differences in ADOS-2 scores calculated based on the new algorithm were investigated through robust $\mathrm{M}$-estimator. There was a significant difference between the groups (Table 2; Figure 2), and post hoc $z$-tests revealed that this significance arose from a significant difference between HC participants and the AN patients ( $z=$ $-4.34, p<0.00)$ and REC patients $(z=-2.56, p=0.028)$. There was no significant difference in the new ADOS- 2 scores between the AN and REC groups $(z=0.1 .92, p=0.134)$ or HC and REC groups $(z=-1.72, p=0.199)$.

\section{Comparing the Original and New Algorithms}

The proportion of participants in each group scoring above the cut-off under the original and new algorithms is presented in Figure 3. A generalized binomial linear mixed effects model showed that there was a significant difference in the number of people meeting cut-off for autism spectrum diagnosis between the original and new algorithms $(b=-5.18, \mathrm{SE}=2.57, z=-2.02$, $p=0.044)$. Under the original algorithm, five HC participants (7.9\%), 13 AN participants (19.7\%), and 7 REC participants (15.2\%) scored above the cut-off of 7. Under the new algorithm, six HC participants (9.5\%), 18 AN participants (27.3\%), and 9 REC participants (19.6\%) scored above the cut-off of 8 .

\section{ADOS and Clinical Characteristics}

Correlations between clinical characteristics and autistic symptomatology assessed with ADOS-2 original and new algorithms can be seen in Table 3 . There was a significant

TABLE 2 | Autism Diagnostic Observation Schedule 2nd Edition (ADOS-2) scores on the original and new algorithms by group.

\begin{tabular}{lcccc}
\hline Measure & $\begin{array}{c}\text { HC } \\
\text { Mean (SD) }\end{array}$ & $\begin{array}{c}\text { AN } \\
\text { Mean (SD) }\end{array}$ & $\begin{array}{c}\text { REC } \\
\text { Mean (SD) }\end{array}$ & $\begin{array}{c}\boldsymbol{F} \text { statistic, } \\
\boldsymbol{p} \text { value }\end{array}$ \\
\hline Original algorithm & $2.14(2.35)$ & $4.06(3.30)$ & $3.28(3.19)$ & $\begin{array}{c}F(2)=6.82, \\
p=0.001\end{array}$ \\
New algorithm & $3.21(2.47)$ & $5.65(3.81)$ & $4.74(4.06)$ & $\begin{array}{c}F(2)=7.97 \\
p<0.001\end{array}$ \\
\hline
\end{tabular}

N.B. HC, healthy control; $A N$, anorexia nervosa; $R E C$, recovered from anorexia nervosa; $S D$, standard deviation.

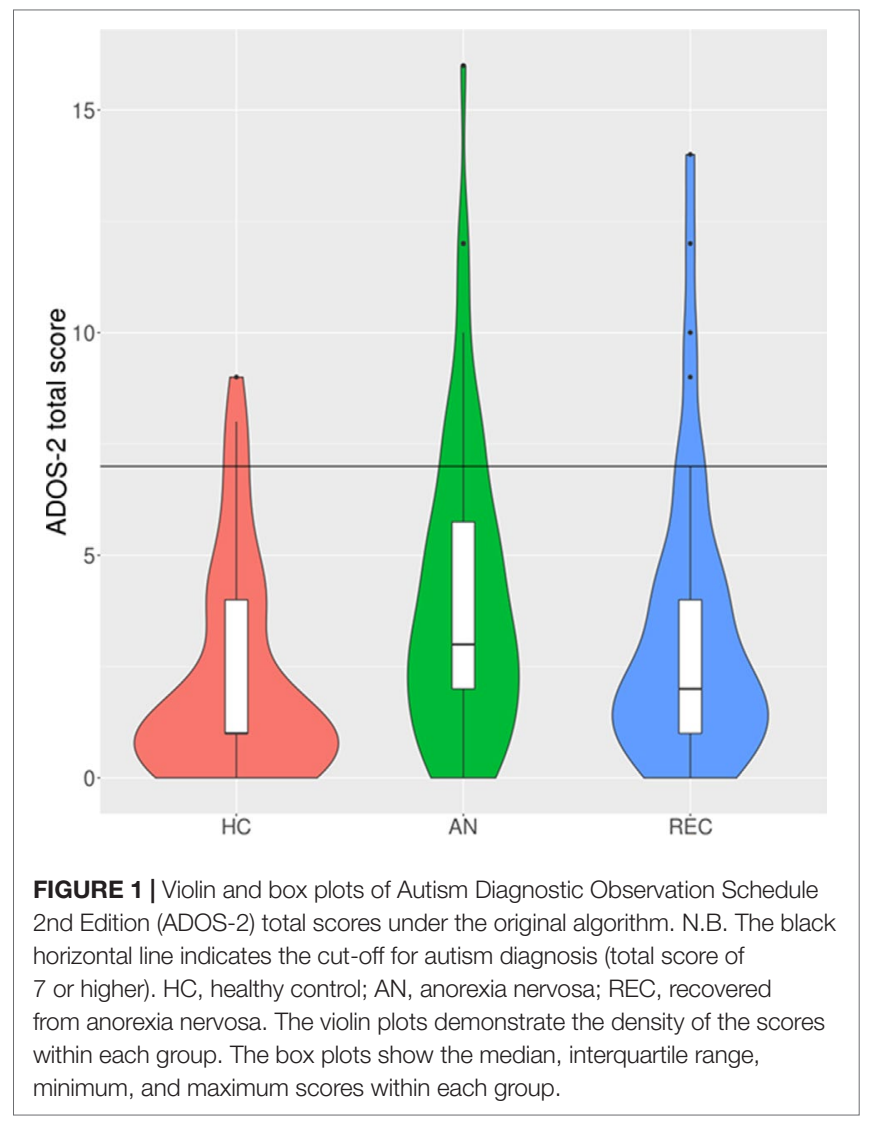

correlation between HADS total score and ADOS-2 scores and between AQ-10 and ADOS-2 scores within the REC group only under the original algorithm. There were no other correlations between the measures within the AN or the REC group that met the corrected $p<0.009$ threshold for significance.

\section{DISCUSSION}

The aim of the present study was to investigate the usefulness of the new ADOS-2 Module 4 algorithm among a highly anxious and depressed group of people with $\mathrm{AN}$ as well as those who had recovered from $\mathrm{AN}$ and people with no history of eating disorders. Importantly, the new ADOS-2 algorithm identifies significantly more currently ill and recovered AN individuals as being on the autism spectrum. The scores from the new algorithm did not correlate significantly with self-reported depression, anxiety, or eating disorder symptomatology. This suggests that the new ADOS-2 algorithm may be useful in identifying AN patients who have co-occurring autism and who may therefore benefit from adapted treatment approaches which account for their possible cognitive and neurodevelopmental differences $(37,38)$.

As hypothesized, there were significantly more AN participants who met cut-off for autism diagnosis than $\mathrm{HC}$ or recovered participants on both the new and the old ADOS-2 algorithms. This echoes previous literature that has documented an overrepresentation of autistic features among those with AN compared to those without AN (21). Although there were no significant 


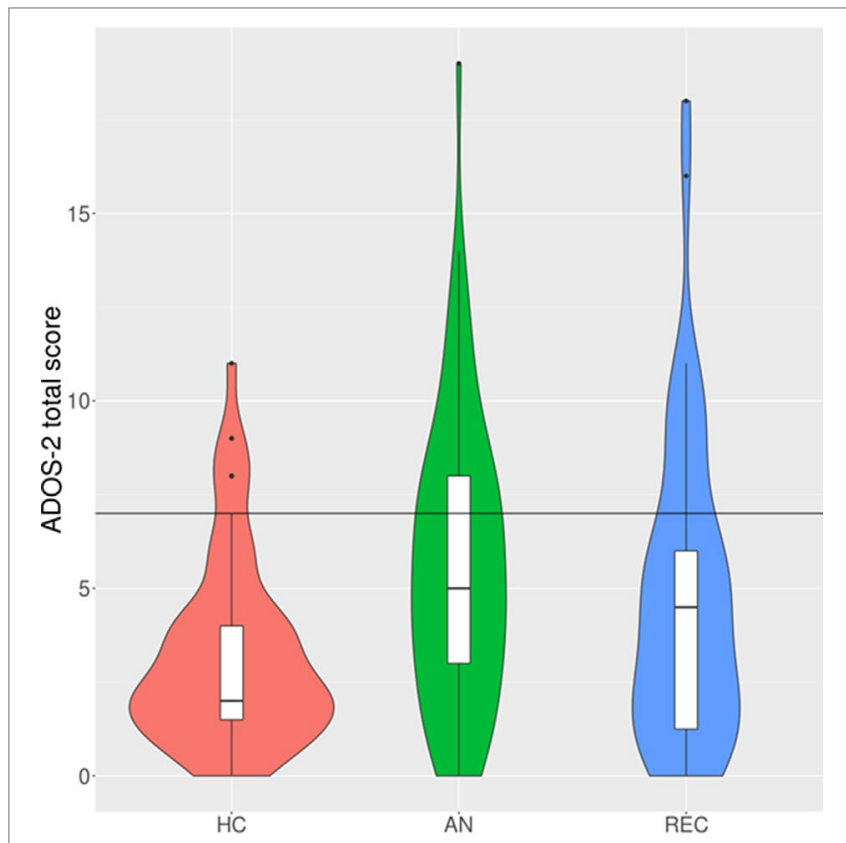

FIGURE 2 | Violin and box plots of ADOS-2 total scores under the new algorithm. N.B. The black horizontal line indicates the cut-off for autism diagnosis (total score of 7 or higher). HC, healthy control; AN, anorexia nervosa; REC, recovered from anorexia nervosa. The violin plots demonstrate the density of the scores within each group. The box plots show the median, interquartile range, minimum, and maximum scores within each group.

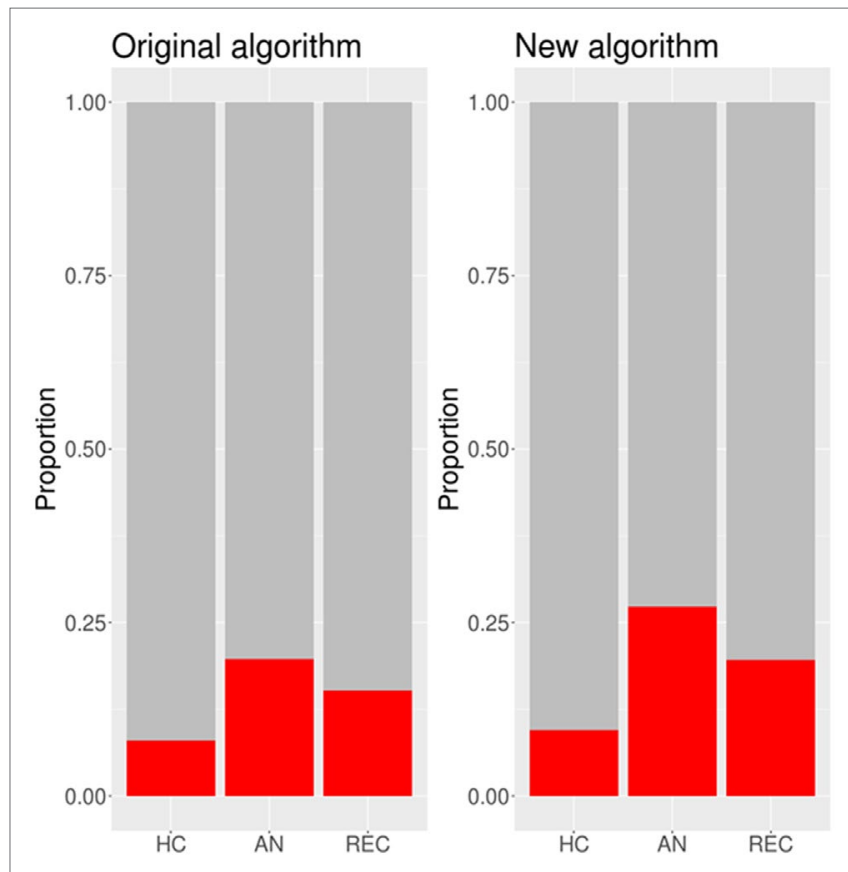

FIGURE 3 | Proportions of participants in each group scoring above the cut-off under the original and new algorithms. N.B. HC, healthy control; AN, anorexia nervosa; REC, recovered from anorexia nervosa. differences between currently ill AN and recovered participants on their ADOS-2 scores, inspection of the median scores per group suggested that recovered participants may represent a midpoint between currently ill AN and HC participants. The present finding could be taken to suggest that rather than having underlying autism, the fact that acutely ill AN patients present with more autistic behaviors may be related to state of illness. However, this is in direct contrast with previous findings showing that similar proportions of participants in currently ill $\mathrm{AN}$ and recovered groups scored above the cut-off on the ADOS-2 (23). Therefore, further longitudinal work is needed to investigate the stability of autistic features through recovery among people with AN.

This interpretation is bolstered by the lack of significant correlations of new algorithm ADOS-2 scores with EDE-Q and BMI. This supports the idea that the new ADOS-2 algorithm is not picking up behaviors associated with anorexia nervosa rather than an underlying autism diagnosis and therefore is not overly sensitive in this population resulting in false-positive autism diagnoses. Furthermore, the fact that the scores on the new ADOS-2 algorithm did not correlate with self-reported depression or anxiety suggests that this algorithm does not erroneously pick up behaviors that may be associated with psychomotor agitation. These findings speak in favor of using the ADOS-2 to assess the presence of autistic features not only among people with eating disorders but also more widely among people with mood and anxiety disorders.

\section{Limitations}

While this paper highlights the usefulness of the new ADOS-2 algorithm among those with AN, it also had some limitations. First, it is important to recognize that regardless of algorithm used, the ADOS-2 alone is not enough to confidently assume that underlying autism is present in AN patients. While the administration of an ADOS-2 with AN patients who may be autistic is an important first step, a full clinical assessment, with elements such as a developmental history, is essential before giving an autism diagnosis. However, if a patient's ADOS-2 scores suggest that autism may be present, it is important to build this into treatment plans as early as possible while waiting for an autism diagnosis appointment, which can take several months.

Second, although the present findings suggest that recovered individuals may represent a midpoint between currently ill AN and HC participants on the ADOS-2 scores, it is important to note that the present study was cross-sectional. Therefore, longitudinal work is needed to further explore the possibility that the presence of autistic features in AN as measured with the ADOS-2 may be related to illness state rather than a reflection of underlying autism. Recognizing the impact of illness state on ADOS-2 score is crucial, as autism is a lifelong diagnosis, and a false diagnosis could negatively affect ED treatment, as many clinicians report having low confidence in their ability to work with an autistic person (39).

Third, the present study assessed the presence of eating disorder symptomatology, mood, and anxiety symptoms using self-report rather than clinical assessments. To confirm that the ADOS-2, in general, and the new algorithm, in particular, do not erroneously pick up psychomotor agitation or other behavior 
TABLE 3 | Correlations between clinical characteristics and ADOS-2 original and new scores in the AN and REC groups.

\begin{tabular}{|c|c|c|c|c|}
\hline \multirow[t]{2}{*}{ Measure } & \multicolumn{2}{|c|}{ Original algorithm } & \multicolumn{2}{|c|}{ New algorithm } \\
\hline & AN & REC & AN & REC \\
\hline BMl & $\rho=-0.25, p=0.044$ & $\rho=-0.05, p=0.748$ & $\rho=-0.15, p=0.217$ & $\rho=0.03, p=0.818$ \\
\hline EDE-Q total & $\rho=0.10, p=0.453$ & $\rho=0.30, p=0.046$ & $\rho=0.11, p=0.420$ & $\rho=0.26, p=0.089$ \\
\hline HADS total & $\rho=0.11, p=0.415$ & $\rho=0.44, p=0.0027$ & $\rho=0.08, p=0.552$ & $\rho=0.32, p=0.032$ \\
\hline$A Q-10$ & $\rho=0.10, p=0.461$ & $\rho=0.42, p=0.005$ & $\rho=0.13, p=0.318$ & $\rho=0.34, p=0.026$ \\
\hline
\end{tabular}

N.B. HC, healthy control; AN, anorexia nervosa; REC, recovered from anorexia nervosa; BMI, body mass index; EDE-Q, Eating disorders examination questionnaire; HADS, Hospital anxiety and depression scale; AQ-10, Autism Quotient (short version).

symptoms related to mental health as unusual sensory interest, replication of the present findings with clinician assessment of mood and anxiety is key. Mislabeling of psychomotor symptoms as autistic features could generate false positive diagnoses that may have a negative impact on illness outcome or treatment. Therefore, further research into the specific nature of these behaviors in those with $\mathrm{AN}$ and potential autism is needed.

Fourth, the present study did not have a comparison group of girls and women with a clinician-confirmed autism diagnosis, who would have given a sense of the changes in ADOS-2 scores generated using the new algorithm with participants who have established autism, and whether the differences in our sample are seen more generally. Furthermore, we did not have a subgroup of participants with AN and clinician-confirmed autism. Inclusion of such a group would be necessary to fully investigate the sensitivity of the new ADOS-2 algorithm in detecting autism within this patient population. Therefore, further research assessing the sensitivity of both the original and new ADOS-2 algorithms under double-blind conditions with autistic and neurotypical participants with and without AN is needed.

Finally, the groups were not exactly matched in size, and significant heteroscedasticity was present in some of the measures. Although steps were taken to conduct robust statistical tests to avoid false findings arising from the presence of heteroscedasticity, future work would benefit from having larger samples sizes and using purposeful matched sampling, especially for recovered individuals.

\section{CONCLUSIONS}

In conclusion, our findings suggest that the new ADOS-2 algorithm is a useful measure of autistic traits among women with current $\mathrm{AN}$ and who have recovered from AN. Our findings suggest that some autistic traits remain after recovery, which may reflect a genuine underlying autism diagnosis. However, the present study

\section{REFERENCES}

1. Gould J, Ashton-Smith J. Missed diagnosis or misdiagnosis? Girls and women on the autism spectrum. Good Autism Practice (GAP) (2011) 12:34-41.

2. American Psychiatric Association. Diagnostic and statistical manual of mental disorders, Fifth edition. Arlington, VA: American Psychiatric Association (2013).

3. Kaland N, Smith L, Mortensen EL. Brief report: cognitive flexibility and focused attention in children and adolescents with Asperger syndrome or is cross-sectional, and the findings need to be confirmed with a longitudinal study of autistic traits before any firm conclusions can be drawn.

\section{ETHICS STATEMENT}

Participants were recruited from a range of clinical and community sites across the UK, under ethical approval from the London Surrey Research Ethics Committee (17/LO/2071; 17/ LO/1960). All participants gave written informed consent before taking part in the study and all procedures were conducted in accordance with the latest version of the Declaration of Helsinki.

\section{AUTHOR CONTRIBUTIONS}

FS carried out recruitment, testing, analysis, and write-up for this research and manuscript. JK-G contributed data and to the write-up of this manuscript. JL carried out recruitment, analysis, and write-up for this research and manuscript. KT led the research group within which this work was conducted and was awarded the funding which enabled it to take place, as well as proofreading the manuscript.

\section{ACKNOWLEDGMENTS}

We would like to thank the girls, women, and families who took part in our research for their time and their clinicians and clinical services for their support in recruitment. We would also like to thank Swiss Anorexia Nervosa Foundation (ref: 58-16) and the MRC and MRF Child and Young Adult Mental Health-Underpinning the Aetiology of Self-Harm and Eating-Disorders (ref: MR/R004595/1) for funding the research and making this study possible. high-functioning autism as measured on the computerized version of the Wisconsin Card Sorting Test. J Autism Dev Disord (2008) 38:1161-5. doi: 10.1007/s10803-007-0474-1

4. Van Eylen L, Boets B, Steyaert J, Evers K, Wagemans J, Noens I. Cognitive flexibility in autism spectrum disorder: explaining the inconsistencies? Res Autism Spectr Disord (2011) 5:1390-401. doi: 10.1016/j.rasd.2011.01.025

5. Gotham K, Bishop SL, Hus V, Huerta M, Lund S, Buja A, et al. Exploring the relationship between anxiety and insistence on sameness in autism spectrum disorders. Autism Res (2013) 6:33-41. doi: 10.1002/aur.1263 
6. Happé F, Frith U. The weak coherence account: detail-focused cognitive style in autism spectrum disorders. J Autism Dev Disord (2006) 36:5-25. doi: 10.1007/s10803-005-0039-0

7. Koldewyn K, Jiang YV, Weigelt $S$, Kanwisher N. Global/local processing in autism: not a disability, but a disinclination. J Autism Dev Disord (2013) 43:2329-40. doi: 10.1007/s10803-013-1777-z

8. Happé F. The weak central coherence account of autism. In: Handbook of Autism and Pervasive Developmental Disorders. Hoboken, NJ, USA: John Wiley \& Sons, Inc. (2005). p. 640-9. doi: 10.1002/9780470939345.ch24

9. Pellicano E, Maybery M, Durkin K, Maley A. Multiple cognitive capabilities/ deficits in children with an autism spectrum disorder: "weak" central coherence and its relationship to theory of mind and executive control. Dev Psychopathol (2006) 18:77-98. doi: 10.1017/S0954579406060056

10. Treasure J, Schmidt U. The cognitive-interpersonal maintenance model of anorexia nervosa revisited: a summary of the evidence for cognitive, socioemotional and interpersonal predisposing and perpetuating factors. $J$ Eat Disord (2013) 1:13. doi: 10.1186/2050-2974-1-13

11. Hodsoll J, Rhind C, Micali N, Hibbs R, Goddard E, Nazar BP, et al. A pilot, multicentre pragmatic randomised trial to explore the impact of carer skills training on carer and patient behaviours: testing the cognitive interpersonal model in adolescent anorexia nervosa. Eur Eat Disord Rev (2017) 25:551-61. doi: $10.1002 /$ erv. 2540

12. Cardi V, Tchanturia K, Treasure J. Premorbid and illness-related social difficulties in eating disorders: an overview of the literature and treatment developments. Curr Neuropharmacol (2018) 16:1122-30. doi: 10.2174/15701 59X16666180118100028

13. Caglar-Nazali HP, Corfield F, Cardi V, Ambwani S, Leppanen J, Olabintan O, et al. A systematic review and meta-analysis of "Systems for Social Processes" in eating disorders. Neurosci Biobehav Rev (2014) 42:55-92. doi: 10.1016/j. neubiorev.2013.12.002

14. Oldershaw A, Lavender T, Sallis H, Stahl D, Schmidt U. Emotion generation and regulation in anorexia nervosa: a systematic review and meta-analysis of self-report data. Clin Psychol Rev (2015) 39:83-95. doi: 10.1016/j.cpr. 2015.04.005

15. Leppanen J, Sedgewick F, Treasure J, Tchanturia K. Differences in the Theory of Mind profiles of patients with anorexia nervosa and individuals on the autism spectrum: a meta-analytic review. Neurosci Biobehav Rev (2018) 90:146-63. doi: 10.1016/j.neubiorev.2018.04.009

16. Tchanturia K, Hambrook D, Curtis H, Jones T, Lounes N, Fenn K, et al. Work and social adjustment in patients with anorexia nervosa. Compr Psychiatry (2013) 54:41-5. doi: 10.1016/j.comppsych.2012.03.014

17. Tchanturia K, Smith E, Weineck F, Fidanboylu E, Kern N, Treasure J, et al. Exploring autistic traits in anorexia: a clinical study. Mol Autism (2013) 4:44. doi: 10.1186/2040-2392-4-44

18. Lang K, Roberts M, Harrison A, Lopez C, Goddard E, Khondoker M, et al. Central coherence in eating disorders: a synthesis of studies using the Rey Osterrieth complex figure test. PLoS One (2016) 11:e0165467. doi: 10.1371/ journal.pone. 0165467

19. Westwood H, Stahl D, Mandy W, Tchanturia K. The set-shifting profiles of anorexia nervosa and autism spectrum disorder using the Wisconsin Card Sorting Test: a systematic review and meta-analysis. Psychol Med (2016) 46:1809-27. doi: 10.1017/S0033291716000581

20. Gillberg C. Autism and anorexia nervosa: related conditions? Nord Psykiatr Tidsskr (1985) 39:307-12. doi: 10.3109/08039488509101911

21. Westwood H, Tchanturia K. Autism spectrum disorder in anorexia nervosa: an updated literature review. Curr Psychiatry Rep (2017) 19:41. doi: 10.1007/ s11920-017-0791-9

22. Lord C, Rutter M, DiLavore P, Risi S, Gotham K, Bishop S. (ADOS-2) Manual (Part 1): Modules 1-4. In: Autism Diagnostic Observation Schedule, 2nd ed. Torrance: CA West Psychol Serv (2012).
23. Bentz M, Jepsen JRM, Pedersen T, Bulik CM, Pedersen L, Pagsberg AK, et al. Impairment of social function in young females with recent-onset anorexia nervosa and recovered individuals. J Adolesc Heal (2017) 60:23-32. doi: 10.1016/j.jadohealth.2016.08.011

24. Keys A, Brožek J, Henschel A, Mickelsen O, Taylor HL. The biology of human starvation. Minneapolis, MN: University of Minnesota Press (1950). 2 vols.

25. Lord C, Risi S, Lambrecht L, Cook EH, Leventhal BL, DiLavore PC, et al. The autism diagnostic observation schedule-generic: a standard measure of social and communication deficits associated with the spectrum of autism. J Autism Dev Disord (2000) 30:205-23. doi: 10.1037/t17256-000

26. Zbozinek TD, Rose RD, Wolitzky-Taylor KB, Sherbourne C, Sullivan G, Stein $\mathrm{MB}$, et al. Diagnostic overlap of generalized anxiety disorder and major depressive disorder in a primary care sample. Depress Anxiety (2012) 29:1065-71. doi: 10.1002/da.22026

27. Allison C, Auyeung B, Baron-Cohen S. Toward brief "red flags" for autism screening: the short autism spectrum quotient and the short quantitative checklist in 1,000 cases and 3,000 controls. J Am Acad Child Adolesc Psychiatry (2012) 51:202-12.e7. doi: 10.1016/j.jaac.2011.11.003

28. Luce KH, Crowther JH. The reliability of the eating disorder examination? Self-report questionnaire version (EDE-Q). Int J Eat Disord (1999) 25: 349-51. doi: 10.1002/(SICI) 1098-108X(199904)25:3<349::AID-EAT15>3.3. CO;2-D

29. Zigmond AS, Snaith RP. The hospital anxiety and depression scale. Acta Psychiatr Scand (1983) 67:361-70. doi: 10.1111/j.1600-0447.1983.tb09716.x

30. Team RC. $R$ : a language and environment for statistical computing. Austria: R Foundation for Statistical Computing (2018).

31. Zeileis A, Hothorn T. Diagnostic checking in regression relationships. (2002).

32. Huber PJ, Ronchetti EM. Robust statistics, ser. Wiley Ser Probab Math Stat New York, NY, USA Wiley-IEEE (1981) 52:54. doi: 10.1002/0471725250

33. Hampel FR, Ronchetti EM, Rousseeuw PJ, Stahel WA. Robust statistics: the approach based on influence functions. John Wiley \& Sons (2011).

34. Venables VN, Ripley BD. Modern applied statistics with S. New York, NY: Springer (2002).

35. Bates D, Mächler M, Bolker B, Walker S. Fitting linear mixed-effects models using lme4. J Stat Softw (2015) 67: (1):48. doi: 10.18637/jss.v067.i01

36. Benjamini Y, Hochberg Y. Controlling the false discovery rate: a practical and powerful approach to multiple testing. J R Stat Soc Ser B (1995) 57:289300. doi: 10.1111/j.2517-6161.1995.tb02031.x

37. Schulte-Rüther M, Mainz V, Fink GR, Herpertz-Dahlmann B, Konrad K. Theory of mind and the brain in anorexia nervosa: relation to treatment outcome. J Am Acad Child Adolesc Psychiatry (2012) 51:832-41.e11. doi: 10.1016/j.jaac.2012.06.007

38. Tchanturia K, Larsson E, Adamson J. How anorexia nervosa patients with high and low autistic traits respond to group Cognitive Remediation Therapy. BMC Psychiatry (2016) 16:334. doi: 10.1186/s12888-016-1044-x

39. Kinnaird E, Norton C, Tchanturia K. Clinicians' views on working with anorexia nervosa and autism spectrum disorder comorbidity: a qualitative study. BMC Psychiatry (2017) 17:292. doi: 10.1186/s12888-017-1455-3

Conflict of Interest Statement: The authors declare that the research was conducted in the absence of any commercial or financial relationships that could be construed as a potential conflict of interest.

Copyright () 2019 Sedgewick, Kerr-Gaffney, Leppanen and Tchanturia. This is an open-access article distributed under the terms of the Creative Commons Attribution License (CC BY). The use, distribution or reproduction in other forums is permitted, provided the original author $(s)$ and the copyright owner(s) are credited and that the original publication in this journal is cited, in accordance with accepted academic practice. No use, distribution or reproduction is permitted which does not comply with these terms. 